\title{
Optimal Investment Strategy for Defined Contribution Pension Scheme under the Heston Volatility Model
}

\author{
Chidi U. Okonkwo', Bright 0. Osu², Silas A. Ihedioha3 ${ }^{3}$ Chigozie Chibuisi ${ }^{4}$ \\ ${ }^{1}$ Department of Mathematics and Statistics, Caritas University, Amorji-Nike, Nigeria \\ ${ }^{2}$ Department of Mathematics, Michael Okpara University of Agriculture, Umudike, Nigeria \\ ${ }^{3}$ Department of Mathematics, Plateau State University, Bokkos, Nigeria \\ ${ }^{4}$ Department of Insurance, University of Jos, Jos, Nigeria \\ Email: chukwuoma99@yahoo.com, osu.bright@mouau.edu.ng, silasihedioha@yahoo.com,chibuisichygoz@yahoo.com
}

How to cite this paper: Okonkwo, C.U., Osu, B.O., Ihedioha, S.A. and Chibuisi, C. (2018) Optimal Investment Strategy for Defined Contribution Pension Scheme under the Heston Volatility Model. Journal of Mathematical Finance, 8, 613-622. https://doi.org/10.4236/jmf.2018.84039

Received: August 27, 2018

Accepted: September 27, 2018

Published: September 30, 2018

Copyright $\odot 2018$ by authors and Scientific Research Publishing Inc. This work is licensed under the Creative Commons Attribution International License (CC BY 4.0).

http://creativecommons.org/licenses/by/4.0/

\begin{abstract}
In this paper, the optimal investment strategy for a defined contribution (DC) pension scheme was modeled with the assumption that the fund is invested partly in riskless assets and partly in risky assets. The market has a constant interest rate, a stochastic volatility that follows the Heston model, the salary is assumed constant over the entire career of the Pension Plan Participant (PPP) and the contribution is a constant proportion of the salary. The CRRA utility function was utilized to obtain a Hamilton-Jacobi-Bellman (HJB) equation. The resulting HJB equation was solved using the Prandtl Asymptotic Matching Method following the works in the literature.
\end{abstract}

\section{Keywords}

Defined Contributory Pension Scheme, Stochastic Volatility, CRRA, Prandtl Asymptotic Matching, Optimal Investment Strategy, HJB

\section{Introduction}

The defined contribution (DC) model is a pension model that has predetermined contribution from the Pension Plan Participant (PPP) and the benefit to the PPP depends on the return on investment of the pension assets. The DC plan is fully funded, privately managed, and there is a third party management of the funds and assets. In this paper, the optimal investment strategies derived with Constant Relative Risk Aversion (CRRA) utility function was considered, volatility was assumed to follow the Heston model with constant interest rate, while 
salary is stochastic. The PPP in the DC pension plan seeks to maximize certain utility function based on his attitude to risk. The most commonly used utility functions are constant relative risk aversion (CRRA), that is, the power or logarithmic utility function, constant absolute risk aversion (CARA), that is, the exponential utility function and hyperbolic absolute risk aversion (HARA) which is a combo utility function since under different assumptions it can transform to the other types. In this work, the CRRA utility function was used.

\section{Theoretical Background}

It is a well known fact that the conditional variance of asset returns, especially stock market returns, is not constant over time [1] [2]. Stock return volatility is serially correlated, and shocks to volatility are negatively correlated with unexpected stock returns. Changes in volatility are persistent [3] [4]. Large negative stock returns tend to be associated with increases in volatility that persist over long periods of time. Stock return volatility appears to be correlated across markets over the world [5].

The groundbreaking papers by Merton [6] opened the floodgates of research on continuous-time portfolio optimization with a constant investment scenario, others have looked at different stochastic scenarios such as stochastic interest rates or stochastic volatility. The investment-consumption problems with the Heston model was considered by [7].

In [8] and [9] optimal control of a defined contribution pension plan where the guarantee depends on the level of interest rates at the fixed retirement date were considered. [10] measured the effect of a minimum interest rate guarantee constraint through the wealth equivalent in case of no constraints and show numerically the guarantees may induce a significant utility loss for relative risk tolerant investors. [11] the most widely used utility function exhibits constant relative risk aversion (CRRA), that is, the power or logarithmic utility function [12] [13] [14] and [15].

\section{The Model}

It is assumed that a PPP who is seeking to maximize the expected utility of his terminal wealth invests in a market made up of risk-free assets and a risky assets. The risky asset is volatile with which can indeed represent the index of the stock market. The dynamics of the underlying assets is given by

$$
\frac{\mathrm{d} B(t)}{B(t)}=r \mathrm{~d} t
$$

for the riskless asset and

$$
\frac{\mathrm{d} S(t)}{S(t)}=\mu \mathrm{d} t+\sqrt{V} \mathrm{~d} W(t)
$$

for the risky asset Where $r$ is a constant risk free interest rate, $\mu(t)$ is the expected growth rate of the risky asset, $\sqrt{V}$ is the volatility of stock with respect 
to the market forces.

The salary process $L(t)$ is assumed to be constant over the entire working career of the PPP. Let $c(t)$ be the contribution rate of the PPP at time $t$, then the contribution process $\psi$ satisfy

$$
\psi(t)=c(t) L(t) \mathrm{d} t
$$

Let $X(t, V)$ be the wealth process of a PPP at time $t$. We assume that the fund administrator invests the contribution in $\eta$ riskless assets and $\pi$ risky assets, then we have the dynamics of the wealth process as

$$
\mathrm{d} X(t, V)=\frac{\eta \mathrm{d} B(t)}{B(t)}+\frac{\pi \mathrm{d} S(t)}{S(t)}+\psi(t)
$$

substituting (1), (2), and (3) in (4) we have

$$
\mathrm{d} X=(X-\pi) r \mathrm{~d} t+\pi(\mu \mathrm{d} t+\sqrt{V} \mathrm{~d} W(t))+c(t) L(t) \mathrm{d} t
$$

simplifying, we have

$$
\mathrm{d} X=(c L(t)+\pi(\mu-r)+X r) \mathrm{d} t+\pi \sqrt{V} \mathrm{~d} W(t)
$$

dividing through by $X$ we have

$$
\frac{\mathrm{d} X}{X}=(c \theta+\Pi(-r+\mu)+r) \mathrm{d} t+\Pi \sqrt{V} \mathrm{~d} W(t)
$$

where $(L(t, V)) / X=\theta$ and $\pi / X=\Pi$. in addition $V(t)$ satisfies the Heston model

$$
\mathrm{d} V_{t}=k(\theta-V) \mathrm{d} t+\xi \sqrt{V} \mathrm{~d} W^{v}(t)
$$

where:

$\theta$ is the long variance at $t \rightarrow \infty$,

$k$ is the rate at which $V_{t}$ reverts to $\theta, \xi$ Is the volatility of volatility and determines the variance of $V_{t}$.

If $2 k \theta>\xi^{2}$ then, $V_{t}$ is strictly positive and is said to satisfy the Feller condition.

$W_{t}$ and $W_{t}^{v}$ are correlated with correlation coefficient $\rho$.

\section{The Optimal Control and Value Function}

\subsection{The Admissible Portfolio Strategy}

The investor will choose the optimal investment and optimal contribution rate that will maximize the expected utility of terminal wealth of the operation. For an arbitrary admissible strategy $f()=.\{\Pi(t, v), c(t, v): t=0\}$. The objective function $G($.) follows:

$$
G(x, v, u)=E[F(T, X, v) \mid X(t)=x, V(t)=v]
$$

For admissible portfolio strategy in stocks $\Pi(t, V), t \in[0, T]$ : we have

$$
E \int_{0}^{T} \Pi(t, V) \Pi(t, V) \mathrm{d} t<\infty
$$

For admissible contribution rate strategy $c(t, V), t \in[0, T]$, we have 


$$
E \int_{0}^{T} c(t, V)^{2} \mathrm{~d} t<\infty
$$

Definition. A strategy $f()=.\{\Pi(t, v), c(t, v): t=0\}$ which is progressively measurable with respect to $\{W(t): 0=s=t\}$ is referred to as an admissible strategy.

Let the collection of all admissible strategies be denoted by $A$. It then follows that the set $A$ can be defined as follows:

$$
\begin{aligned}
& \mathcal{A}=f(t, V)=\left\{(\Pi(t, V), C(t, V)) \in R^{n} \times R\right\} \\
& E \int_{0}^{T} \Pi(t, V) \Pi(t, V) \mathrm{d} t<\infty ; E \int_{0}^{T} c(t, V)^{2} \mathrm{~d} t<\infty
\end{aligned}
$$

\subsection{The Value Function}

Let the value function be defined as

$$
\max _{\Pi, C} E\left[\frac{\alpha \mathrm{e}^{-\beta t} C^{1-\gamma} \mathrm{d} t}{1-\gamma}+\frac{(1-\alpha) \alpha \mathrm{e}^{-\beta t} X^{1-\gamma}}{1-\gamma}\right]
$$

$X_{T}$ is the value at time $T$ of a trading strategy that finances $\left\{C_{t}\right\}_{t=0}^{T}$.

$\gamma$ is the risk aversion coefficients.

$\beta$ is the subjective discount rate.

$\alpha$ determines the relative importance of the intermediate consumption when $\alpha=0$, expected utility only depends on the terminal wealth.

The problem confronting the PPP at time $t$ is to select the portfolio weights and contribution rate processes $\{\Pi(t, v), c(t, v)\}_{t s T}$ that maximize the expected utility of terminal wealth of the investor subject to:

$$
\left\{\begin{array}{l}
\frac{\mathrm{d} X}{X}=(c \theta+\Pi(-r+\mu)+r) \mathrm{d} t+\Pi \sqrt{V} \mathrm{~d} W(t) \\
\mathrm{d} V_{t}=k\left(\theta-V_{t}\right) \mathrm{d} t+\xi \sqrt{V_{t}} \mathrm{~d} W_{t}^{v}
\end{array}\right.
$$

We now consider an investor that chooses power utility function. By applying stochastic dynamic programming approach and Ito Lemma our Hamilton-JacobiBellman equation characterized by the optimal solutions to the problem of the investor becomes [5]

$$
\begin{aligned}
& \frac{\partial}{\partial t} H(t, X, V)+\frac{1}{2} X^{2} \Pi^{2} \sqrt{V} \sqrt{V} \frac{\partial^{2}}{\partial X^{2}} H(t, \Pi, V) \\
& +X(\Pi(-r+\mu)+r) \frac{\partial}{\partial X} H(t, X, V)+C \frac{\partial}{\partial X} H(t, X, V) \\
& +X \Pi \sqrt{V} \rho \xi \sqrt{V} \frac{\partial^{2}}{\partial X \partial V} H(t, X, V)+\frac{1}{2} \xi^{2} \sqrt{V}^{2} \frac{\partial^{2}}{\partial V^{2}} H(t, X, V) \\
& +k(\theta-V) \frac{\partial}{\partial V} H(t, X, V)+\frac{\alpha \mathrm{e}^{-\beta t} C^{1-\gamma}}{1-\gamma}=0
\end{aligned}
$$

With boundary condition

$$
H(t, X, V)=(1-\alpha) \frac{X^{1-\gamma}}{1-\gamma}
$$

$H$ is conjectured to have a solution of the form 


$$
H(t, X, V)=\mathrm{e}^{-B t} \frac{X^{1-\gamma}}{1-\gamma}[F(X, t)]^{\gamma}
$$

where $\frac{\partial}{\partial t} H(t, X, V), \frac{\partial}{\partial X} H(t, X, V), \frac{\partial^{2}}{\partial X^{2}} H(t, X, V)$ and $\frac{\partial^{2}}{\partial V^{2}} H(t, X, V)$ are the partial derivatives with respect to $t, X$ and $V$.

With the above assumptions and the fact that contribution cannot be negative, then the optimal values of the portfolio weight and contributions are:

$$
\Pi^{*}=\frac{V \rho \xi \gamma \frac{\partial}{\partial V} F(t, V)-F(t, V)(r-\mu)}{V \gamma F(t, V)}
$$

and

$$
C^{*}=-\frac{X \alpha^{\frac{1}{\gamma}}}{F(t, V)}
$$

Substituting the derivatives as well as the optimal values in (10) resultsin the HJB Equation (7)

$$
\begin{aligned}
& -\frac{\partial}{\partial t} F(t, V)+\vartheta_{1} V \frac{\partial^{2}}{\partial V^{2}} F(t, V)+\left(\vartheta_{2} V+\vartheta_{3}\right) \frac{\partial}{\partial V} F(t, V) \\
& +\frac{\vartheta_{4} V\left(\frac{\partial}{\partial V} F(t, V)\right)^{2}}{F(t, V)}+\left(\vartheta_{5} V+\vartheta_{6}\right) F(t, V)+\alpha^{\frac{1}{\gamma}}=0
\end{aligned}
$$

with boundary conditions

$$
F(0, V)=(1-\alpha)^{\frac{1}{\gamma}}
$$

\section{Solving the PDE}

The HJB PDE so derived has no closed form solution, this is mainly as a result of the presence the non homogeneous terms $\alpha^{\frac{1}{\gamma}} F_{V V}$ and $\frac{F_{V}^{2}}{F}$ in (13).

A closed-form approximate solution for the PDE was derived by Zhang and Ge [7]. This they did by using the Prandtl's assymptotic matching method. the trick is, first remove the constant term $\alpha^{\frac{1}{\gamma}}$ solve the resulting PDE and call the solution $F_{1}$. Thus $F_{1}$ satisfy the PDE

$$
\begin{aligned}
& -\frac{\partial}{\partial t} F(t, V)+\vartheta_{1} V \frac{\partial^{2}}{\partial V^{2}} F(t, V)+\left(V \vartheta_{2}+\vartheta_{3}\right) \frac{\partial}{\partial V} F(t, V) \\
& +\frac{\vartheta_{4} V\left(\frac{\partial}{\partial V} F(t, V)\right)^{2}}{F(t, V)}+\left(V \vartheta_{5}+\vartheta_{6}\right) F(t, V)=0
\end{aligned}
$$

The second step is to remove the two non linear terms while retaining the non homogeneous term, solve the resulting PDE and call the solution $F_{2}$, thus $F_{2}$ satisfy the PDE

$$
-\frac{\partial}{\partial t} F(t, V)+\left(V \vartheta_{2}+\vartheta_{3}\right) \frac{\partial}{\partial V} F(t, V)+\left(V \vartheta_{5}+\vartheta_{6}\right) F(t, V)+\alpha^{\gamma^{-1}}=0
$$


The third step is to factor out the common terms in $F_{1}$ and $F_{2}$ and call that $F_{3}$. The solution of the original HJB equation will then be given by

$$
F=F_{1}(t, V)+F_{2}(t, V)-F_{3}(t, V)
$$

Following [5] and [16], $F_{1}$ is

$$
F_{1}(t, V)=(1-\alpha)^{\gamma^{-1}} \mathrm{e}^{\psi_{1}(t) V+\psi_{2}(t)}
$$

where

$$
\psi_{1}= \begin{cases}(1-\alpha)^{\gamma^{-1}} \zeta_{1}+\zeta_{2} & a_{2} \neq 0 \\ (1-\alpha)^{\gamma^{-1}} \zeta_{3}+\zeta_{4} & a_{2}=0\end{cases}
$$

and

$$
\begin{aligned}
\psi_{2}= & \begin{array}{ll}
-\frac{1}{2} \frac{a_{3} \ln \left(\left|\left(a_{2}+\sqrt{\Phi}\right) e^{-\sqrt{\Phi} \tau}-a_{2}+\sqrt{\Phi}\right|\right)}{a_{7} \sqrt{|\Phi|}}+\left(m-\frac{1}{2} \frac{a_{3} \sqrt{\Phi}}{a_{7}}\right) t & \Phi>0 \\
-\frac{a_{3} \ln \left(\left|\frac{1}{2} a_{2} t-1\right|\right)}{a_{7}}+m t & \Phi=0 a_{1} \\
-\frac{a_{3}}{a_{7}} \ln ||-\cos \left(\frac{1}{2} \sqrt{-\Phi} t\right)+\frac{\left.\sin \left(\frac{1}{2} \sqrt{-\Phi} t\right) a_{2} \mid\right)}{\sqrt{-\Phi}} \mid+m t & \Phi<0 \\
= & -\frac{1}{2} \xi^{2} a_{2}=\frac{\rho \xi A(\gamma-1)}{\gamma}-k a_{3}=\theta k a_{4}=\frac{1}{2} \xi^{2}\left(-\rho^{2}+1\right)(\gamma-1) a_{5}
\end{array} \\
= & \frac{1}{2} \frac{(1-\gamma) A^{2}}{\gamma^{2}} a_{6}=-\frac{\beta}{\gamma}+\frac{(1-\gamma) r}{\gamma}
\end{aligned}
$$

with

$$
\begin{gathered}
\zeta_{1}=\mathrm{e}^{a_{2} b_{1} t+b_{2}(V)\left(\mathrm{e}^{a_{2} t}-1\right)}, \quad \zeta_{2}=\frac{\alpha^{\gamma^{-1}}\left(\Gamma\left(b_{1}, b_{2}(V)\right)-\Gamma\left(b_{1}, b_{2}(V) \mathrm{e}^{a_{2} t}\right)\right)}{a_{2}\left(-b_{2}(V)\right)^{b_{1}} \mathrm{e}^{b_{2}(V)}}, \\
\zeta_{3}=\mathrm{e}^{2 b_{3} b_{4}(V) t+b_{3}^{2} t^{2}} \text { and } \zeta_{4}=\frac{1}{2} \frac{\alpha^{\gamma^{-1}} \sqrt{\pi}\left(\operatorname{erfi}\left(b_{3} t+b_{4}(V)\right)-\operatorname{erfi}\left(b_{4}(V)\right)\right)}{b_{3} \mathrm{e}^{\left(b_{4}(V)\right)^{2}}}
\end{gathered}
$$

$F_{2}$ is given by

$$
F_{2}= \begin{cases}(1-\alpha)^{\gamma^{-1}} v_{1}+\alpha^{\gamma^{-1}} v_{2} & a_{2} \neq 0 \\ (1-\alpha)^{\gamma^{-1}} v_{3}+\alpha^{\gamma^{-1}} v_{4} & a_{2}=0\end{cases}
$$

where

$$
\begin{gathered}
v_{1}=\mathrm{e}^{a_{2} b_{1} t+b_{2}(V) \mathrm{e}^{a_{2} t}-1}, \quad v_{2}=\frac{\Gamma\left(b_{1}, b_{2}(V)\right)-\Gamma\left(b_{1}, b_{2}(V) \mathrm{e}^{a_{2} t}\right)}{a_{2}\left(-b_{2}(V)\right)^{b_{1}} \mathrm{e}^{b_{2}(V)}}, \\
v_{3}=\mathrm{e}^{2 b_{3} b_{4}(V) t+b_{3}^{2} t^{2}} \text { and } v_{4}=\frac{1}{2} \frac{\sqrt{\pi}\left(\operatorname{erfi}\left(b_{3} t+b_{4}(V)\right)-\operatorname{erfi}\left(b_{4}(V)\right)\right)}{b_{3} \mathrm{e}^{\left(b_{4}(V)\right)^{2}}}
\end{gathered}
$$


$F_{3}$ then becomes

$$
F_{3}= \begin{cases}\frac{(1-\alpha)^{\gamma^{-1}} a_{5} \mathrm{e}^{a_{2} b_{1} t+b_{2}(V)\left(\mathrm{e}^{a_{2} t}-1\right)}}{a_{2}\left(\mathrm{e}^{a_{2} t}-1\right)} & a_{2} \neq 0 \\ (1-\alpha)^{\gamma^{-1}} \mathrm{e}^{2 b_{3} b_{4}(V) t+b_{3}^{2} t^{2}} & a_{2}=0\end{cases}
$$

with

$$
\begin{gathered}
b_{1}=\frac{a_{2} a_{6}-a_{3} a_{5}}{a_{2}^{2}}, \quad b_{2}(V)=\frac{a_{2} a_{5}-a_{3} a_{5}}{a_{2}^{2}}, \\
b_{3}=\frac{\sqrt{2}}{2} \sqrt{a_{3} a_{5}} \text { and } b_{4}=\frac{1}{2} \frac{\sqrt{2}\left(a_{5} V+a_{6}\right)}{\sqrt{a_{3} a_{5}}} m=a_{6}-\frac{1}{2} \frac{a_{3} a_{2}}{a_{7}} .
\end{gathered}
$$

The derivatives are given by

$$
F_{1_{V}}(t, V)=(1-\alpha)^{\gamma^{-1}} \mathrm{e}^{\psi_{1}(t) V+\psi_{2}(t)} \psi_{1}(t)
$$

with

$$
\begin{aligned}
& \eta_{1}=\frac{a_{5} \mathrm{e}^{a_{2} b_{1} t+b_{2}(v)\left(\mathrm{e}^{a_{2} t}-1\right)}}{a_{2}\left(\mathrm{e}^{a_{2} t}-1\right)}, \quad \eta_{2}=\frac{a_{5}\left(\Gamma\left(b_{1}, 1\right)-\Gamma\left(b_{1}, \mathrm{e}^{a_{2} t}\right)\right)}{a_{2}^{2}\left(-b_{2}(v)\right)^{b_{1}} \mathrm{e}^{b_{2}(v)}}\left(1+\frac{b_{1}}{b_{2}(v)}\right), \\
& \eta_{3}=\mathrm{e}^{2 b_{3} b_{4}(V) t+b_{3}^{2} t^{2}} \text { and } \eta_{4}=a_{3}^{-1}+\frac{\sqrt{\pi} b_{4}(V)\left(\operatorname{erfi}\left(b_{3} t+b_{4}(v)\right)-\operatorname{erfi}\left(b_{4}(v)\right)\right)}{a_{3} \mathrm{e}^{\left(b_{4}(v)\right)^{2}}} \\
& F_{2_{V}}= \begin{cases}(1-\alpha)^{\gamma^{-1}} \eta_{1}-\alpha^{\gamma^{-1}} \eta_{2} & a_{2} \neq 0 \\
(1-\alpha)^{\gamma^{-1}} \eta_{3}+\alpha^{\gamma^{-1}} \eta_{4} & a_{2}=0\end{cases} \\
& F_{3_{V}}= \begin{cases}\frac{(1-\alpha)^{\gamma^{-1}} a_{5}\left(e\left(a_{2} t\right)-1\right) \mathrm{e}^{a_{2} b_{1} t+b_{2}(v)\left(\mathrm{e}^{\left(a_{2} t\right.}-1\right)} a_{2}}{a_{2}\left(\mathrm{e}^{a_{2} t}-1\right)} & a_{2} \neq 0 \\
(1-\alpha)^{\gamma^{-1}} \mathrm{e}^{2 b_{3} b_{4}(V) t+b_{3}^{2} t^{2}} & a_{2}=0\end{cases} \\
& F_{V}(t, V)=F_{1_{V}}(t, V)+F_{2_{V}}(t, V)-F_{3_{V}}(t, V) \\
& \Pi^{*}=\rho \xi \frac{F_{V}}{F}-\frac{r-\mu}{V \gamma}
\end{aligned}
$$

and

$$
\begin{gathered}
C^{*}=\frac{X \alpha^{\frac{1}{\gamma}}}{F_{1}+F_{2}-F_{3}} \\
\Pi^{*}=\rho \xi \frac{F_{1_{V}}+F_{2_{V}}-F_{3_{V}}}{F_{1}+F_{2}-F_{3}}-\frac{r-\mu}{V \gamma}
\end{gathered}
$$

\section{Optimal Investment Policy}

Proposition 1. The optimal stock investment policy with the portfolio weight $\Pi$ is given as (17), (18) and (19) 


$$
\Pi^{*}=\rho \xi \frac{F_{1_{V}}+F_{2_{V}}-F_{3_{V}}}{F_{1}+F_{2}-F_{3}}-\frac{r-\mu}{V \gamma}
$$

Proposition 2. The optimal contribution rate of a PPP strategy is

$$
C^{*}=\frac{X \alpha^{\frac{1}{\gamma}}}{F_{1}+F_{2}-F_{3}}
$$

with $F$ and $F_{V}$ given by (17), (18), (19), (20), (21), and (22)

From proposition (1) and (2) it follows that for optimality of the stock investment and contribution to exist, the following must hold:

1) $F_{1}+F_{2} \neq F_{3}$ 2) $\gamma \neq 0$. In addition, for $\Pi^{*}$ to be positive, then, $\rho, \xi$ and $F_{1_{V}}+F_{2_{V}}-F_{3_{V}}$ must not be 0, else $\mu>r$.

The $3 \mathrm{D}$ plot for the optimal contribution and optimal stock allocations are given in Figure 1(a) and Figure 1(b) respectively using the following parameters; $\alpha=0$,

$$
A=0.5, \beta=1, \gamma=0.5, \xi=0.5, \rho=-0.5, k=10, r=0.05, \theta=0.05
$$

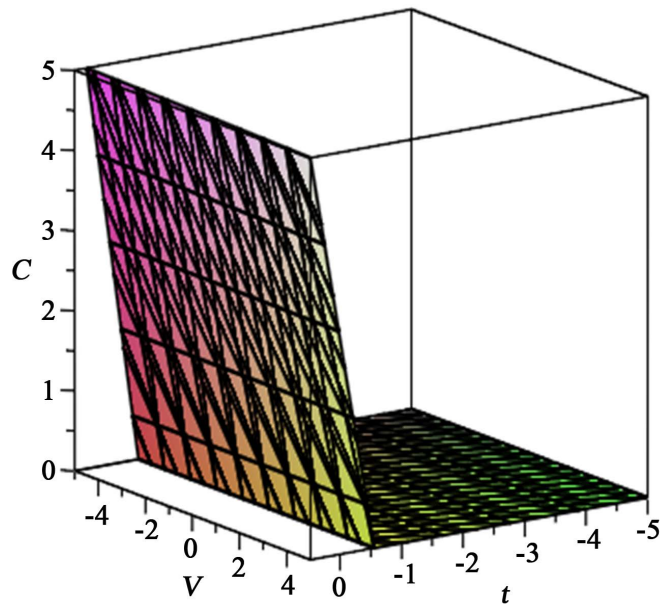

(a)

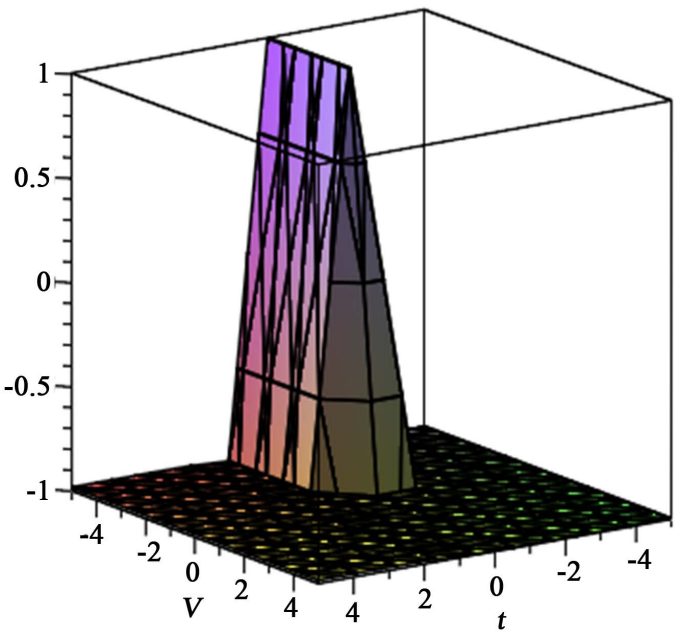

(b)

Figure 1. (a) Optimal contribution; (b) Optimal stock allocation. 


\section{Conclusion}

This paper considered the optimal investment problem for a pension plan participants in a defined contribution (DC) plan. It was assumed that the interest rate and salary are constant over time and stock price follows a Heston volatility model. The HJB equation was obtained and solved assuming that the CRRA utility function and following the works of [5] and [16].

\section{Acknowledgements}

Manifold thanks to the reviewers.

\section{Conflicts of Interest}

The authors declare no conflicts of interest regarding the publication of this paper.

\section{References}

[1] Bollerslev, T., Chou, R.Y. and Kroner, K. (1992) ARCH Modeling in Finance. Journal of Econometrics, 52, 5-59. https://doi.org/10.1016/0304-4076(92)90064-X

[2] Campbell, J.Y., Cocco, J., Gomes, F., Maenhout, P.J. and Viceira, L.M. (2001) Stock Market Mean Reversion and the Optimal Equity Allocation of a Long-Lived Investor. European Finance Review, 5, 269-292. https://doi.org/10.1023/A:1013860504885

[3] French, K.R., Schwert, G.W. and Stambaugh, R.F. (1987) Expected Stock Returns and Volatility. Journal of Financial Economics, 19, 3-29.

[4] Campbell, J.Y. and Hentschel, L. (1992) No News Is Good News. An Asymmetric Model of Changing Volatility in Stock Returns. Journal of Financial Economics, 31, 281-318.

[5] Liu, J. (2007) Portfolio Selection in Stochastic Environments. The Review of Financial Studies, 20, 1-39.

[6] Merton, R.C. (1969) Lifetime Portfolio Selection under Uncertainty: The Continuous-Time Case. The Review of Economics and Statistics, 51, 247-257. https://doi.org/10.2307/1926560

[7] Fleming, W.H. and Hernandez-Hernandez, D. (2003) An Optimal Consumption Model with Stochastic Volatility. Finance Stochast, 7, 245-262. https://doi.org/10.1007/s007800200083

[8] Boulier, J.-F., Huang, S. and Taillard, G. (2001) Optimal Management under Stochastic Interest Rates: The Case of a Protected Defined Contribution Pension Fund. Insurance, 28, 173-189.

[9] Deelstra, G., Grasselli, M. and Koehl, P.-F. (2003) Optimal Investment Strategies in the Presence of a Minimum Guarantee. Insurance, 33, 189-207.

[10] Jensen, B.A. and Sørensen, C. (1999) Paying for Minimum Interest Guarantees. Who Should Compensate Who? European Financial Management, 7, 183-211. https://doi.org/10.1111/1468-036X.00152

[11] Boulier, J.-F., Huang, S.J. and Taillard, G. (2001) Optimal Management under Stochastic Interest Rates: The Case of a Protected Defined Contribution Pension Fund. Insurance: Mathematics and Economics, 28, 173-189. https://doi.org/10.1016/S0167-6687(00)00073-1 
[12] Cairns, A.J.G., Blake, D. and Dowd, K. (2006) Stochastic Lifestyling: Optimal Dynamic Asset Allocation for Defined Contribution Pension Plans. Journal of Economic Dynamics \& Control, 30, 843-877. https://doi.org/10.1016/j.jedc.2005.03.009

[13] Gao, J. (2008) Stochastic Optimal Control of DC Pension Funds. Insurance, 42, 1159-1164.

[14] Nkeki, C.I. (2011) On Optimal Portfolio Management of the Accumulation Phase of a Defined Contributory Pension Scheme. University of Ibadan, Ibadan.

[15] Deelstra, G., Grasselli, M. and Koehl, P.-F. (2003) Optimal Investment Strategies in the Presence of a Minimum Guarantee. Insurance, 33, 189-207.

[16] Zhang, Q. and Ge, L. (2016) Optimal Strategies for Asset Allocation and Consumption under Stochastic Volatility. Applied Mathematics Letters. 